\title{
CHANDRA OBSERVATIONS OF COMETS 8P/TUTTLE AND 17P/HOLMES DURING SOLAR MINIMUM
}

\author{
D. J. Christian ${ }^{1,2,9}$, D. Bodewits ${ }^{3,10}$, C. M. Lisse $^{4}$, K. Dennerl ${ }^{5}$, S. J. Wolk ${ }^{6}$, H. Hsieh $^{7}$, T. H. Zurbuchen $^{8}$, And L. Zhao ${ }^{8}$ \\ ${ }^{1}$ Eureka Scientific, 2420 Delmer Ave, Suite 100, Oakland, CA, 94602, USA; damian.christ@ gmail.com, damian.christian@csun.edu \\ ${ }^{2}$ Department of Physics and Astronomy, California State University Northridge, 18111 Nordoff Street, Northridge, CA 91330, USA \\ ${ }^{3}$ NASA Goddard Space Flight Center, Solar System Exploration Division, Mailstop 690.3, Greenbelt, MD 20771, USA; Dennis.Bodewits@ nasa.gov \\ ${ }^{4}$ Planetary Exploration Group, Space Department, Johns Hopkins University Applied Physics Laboratory, 11100 Johns Hopkins Rd, Laurel, MD 20723, USA; \\ carey.lisse@jhuapl.edu \\ ${ }^{5}$ Max-Planck-Institut für extraterrestrische Physik, Postfach 1603, 85740 Garching, Germany; kod@mpe.mpg.de \\ ${ }^{6}$ Chandra X-Ray Center, Harvard-Smithsonian Center for Astrophysics, 60 Garden Street, Cambridge, MA 02138, USA; swolk@cfa.harvard.edu \\ 7 Department of Physics and Astronomy, Queen's University Belfast, Astronomy Research Centre, Belfast, UK; h.hseih@qub.c.uk \\ ${ }^{8}$ Department of Atmospheric Oceanic and Space Sciences, University of Michigan, 2455 Hayward Street, Ann Arbor, MI 48109-2143, USA; thomasz@umich.edu, \\ lzh@umich.edu \\ Received 2009 April 26; accepted 2010 February 11; published 2010 March 25
}

\begin{abstract}
We present results for Chandra X-ray Observatory observations of two comets made during the minimum of solar cycle 24 . The two comets, $17 \mathrm{P} /$ Holmes (17P) and 8P/Tuttle (8P), were very different in their activity and geometry. $17 \mathrm{P}$ was observed, for $30 \mathrm{ks}$ right after its major outburst, on 2007 October 31 (10:07 UT), and comet 8P/Tuttle was observed in 2008 January for $47 \mathrm{ks}$. During the two Chandra observations, 17P was producing at least 100 times more water than $8 \mathrm{P}$ but was 2.2 times further away from the Sun. Also, $17 \mathrm{P}$ was at a relatively high solar latitude $(+19.1)$ while $8 \mathrm{P}$ was observed at a lower solar latitude (3:4). The $\mathrm{X}$-ray spectrum of $17 \mathrm{P}$ is unusually soft with little significant emission at energies above $500 \mathrm{eV}$. Depending on our choice of background, we derive a 300-1000 eV flux of $0.5-4.5 \times 10^{-13} \mathrm{erg} \mathrm{cm}^{-2} \mathrm{~s}^{-1}$, with over $90 \%$ of the emission in the $300-400 \mathrm{eV}$ range. This corresponds to an X-ray luminosity between 0.4 and $3.3 \times 10^{15} \mathrm{erg} \mathrm{s}^{-1}$. However, we cannot distinguish between this significant excess emission and possible instrumental effects, such as incomplete charge transfer across the CCD. 17P is the first comet observed at high latitude during solar minimum. Its lack of X-rays in the $400-1000 \mathrm{eV}$ range, in a simple picture, may be attributed to the polar solar wind, which is depleted in highly charged ions. 8P/Tuttle was much brighter, with an average count rate of 0.20 counts s$^{-1}$ in the $300-1000 \mathrm{eV}$ range. We derive an average $\mathrm{X}$-ray flux in this range of $9.4 \times 10^{-13} \mathrm{erg} \mathrm{cm}^{-2} \mathrm{~s}^{-1}$ and an X-ray luminosity for the comet of $1.7 \times 10^{14} \mathrm{erg} \mathrm{s}^{-1}$. The light curve showed a dramatic decrease in flux of over $60 \%$ between observations on January 1 and 4 . When comparing outer regions of the coma to inner regions, its spectra showed a decrease in ratios of $\mathrm{C} \mathrm{VI} / \mathrm{C} \mathrm{v}, \mathrm{O}$ vIII/ $\mathrm{O}$ vII, as predicted by recent solar wind charge exchange (SWCX) emission models. There are remarkable differences between the X-ray emission from these two comets, further demonstrating the qualities of cometary X-ray observations, and SWCX emission in general as a means of remote diagnostics of the interaction of astrophysical plasmas.
\end{abstract}

Key words: comets: individual (Comet 8P/Tuttle, Comet 17P/Holmes) - solar wind - techniques: spectroscopic $\mathrm{X}$-rays: general

Online-only material: color figures

\section{INTRODUCTION}

Comets in the inner solar system are generally bright $\mathrm{X}$-ray emitters in the soft $\mathrm{X}$-ray band $(0.2-1.0 \mathrm{keV})$ with a total $\mathrm{X}$-ray emitting power of 0.2-1.0 GW (Dennerl et al. 1997). The X-ray emission is driven by charge exchange between highly charged solar wind ions and the neutral gas in the coma (Cravens 1997; Krasnopolsky et al. 1997). The charge exchange process is a quasi-resonant process, implying that the resulting emission is strongly dependent on properties of both the neutral gas (i.e., the comet) and the solar wind (Schwadron \& Cravens 2000; Kharchenko \& Dalgarno 2000, 2001; Beiersdorfer et al. 2003; Kharchenko et al. 2003; Krasnopolsky 2004, 2006; Bodewits et al. 2004, 2006, 2007).

A recent survey of all comets thus far observed with Chandra (Bodewits et al. 2007) demonstrated that the X-ray spectrum mainly reflects the state of the local solar wind during the

\footnotetext{
9 Author to whom any correspondence should be addressed.

${ }^{10}$ NASA Postdoctoral fellow.
}

observations. During solar minimum, the solar wind can be considered to be in a bimodal state, with a slow, highly variable wind within roughly $15^{\circ}$ of the Sun's equator, and a fast, tenuous and more steady polar wind at higher latitudes (McComas et al. 2003). These winds originate in different parts of the solar atmosphere, and the different temperatures at these source regions imply different charge balances. The slow equatorial wind stems from hotter streamer belt regions than the polar wind that originates in polar coronal holes, and ions in the equatorial wind are on average in a higher ionization state than ions in the polar wind. During solar maximum, this bimodality disappears, leaving the solar wind in a highly chaotic state. As soon as charge exchange was acknowledged to be the driving process behind the cometary X-rays (Cravens 1997; Dennerl et al. 1997; Krasnopolsky et al. 1997; Lisse et al. 2001), it was predicted that the bimodality of the solar wind should be reflected in cometary X-ray spectra (Schwadron \& Cravens 2000; Kharchenko \& Dalgarno 2001; Bodewits et al. 2004).

Two comparative reviews of all comets observed with Chandra in the period 2001-2006 (Bodewits et al. 2007; 
Krasnopolsky 2006) linked cometary spectra to solar wind states by comparing the low-energy X-ray flux attributed to carbon and nitrogen ions with the higher energy X-ray flux attributed to oxygen ions. The derived ratio between the abundances of bare and H-like oxygen ions (before charge exchange) was linked to solar wind freezing-in temperatures, showing that the Chandra comet sample covered a continuous range of solar wind states. Interestingly enough, the comets observed at high latitude were found to be interacting with hot, highly ionized solar winds. As these observations were all performed around 2001, these hot spectra could be linked to coronal mass ejections (CMEs) occurring around solar maximum. This led to the conclusion that Chandra had not yet observed a comet interacting with the cold, polar wind.

In this paper, we present Chandra observations of two Jupiter family comets made during solar minimum: comet 17P/ Holmes (hereafter 17P) and comet 8P/Tuttle (hereafter 8P). The observations of $17 \mathrm{P}$ were extraordinary due to both the state and the geometrical circumstances of the comet. Holmes is a Jupiter family comet that underwent an unexpected major outburst that started around 2007 October $23.7 \pm 0.2$ (Schleicher 2007; Gaillard et al. 2007; Sekanina 2008). While at a heliocentric distance of 2.44 AU and at 1.63 AU from Earth, the comet's visual brightness increased from $m_{v}=17$ to $m_{v}=2.5$. Whatever caused this outburst is as yet unclear, but it released vast amounts of gas and dust (Montalto et al. 2008). According to SWAN observations by Combi et al. (2008), the water production reached a maximum of $1.4 \times 10^{30} \mathrm{~mol} \mathrm{~s}^{-1}$ on October 27 , and then decreased steadily in the weeks after. The comet remained a bright object on the sky for months after the outburst, mainly due to its exceptionally high dust-to-gas ratio. Chandra observed the comet a week after its outburst, on October 31. Observations done by Salyk et al. (2007) and Schleicher (2007) on the days before and after the observations indicate that around the time of our observations, the comet had a gas production rate between $3-5 \times 10^{29} \mathrm{~mol} \mathrm{~s}^{-1} .17 \mathrm{P}$ thus had the largest gas production rate and heliocentric distance of any comet thus far observed with Chandra, and is the first comet that was observed with Chandra at high solar latitude $\left(+199^{\circ}\right)$ during solar minimum.

Comet 8P/Tuttle is a comet from the Oort cloud reservoir now in a short-period Halley-type orbit, which has been suggested to be a contact binary (Harmon et al. 2008; Lamy et al. 2008). It was observed as it passed within 0.25 AU of Earth in 2008 January. 8P orbits the Sun with a period of 13.5 years with a high inclination $\left(I=55^{\circ}\right)$, but during the Chandra observations on 2008 January $1-4$ its heliographic latitude was $1^{\circ}-4^{\circ}$, i.e., very close to the Sun's equatorial plane. Water production rates were between $(2.1-2.4 \pm 0.1) \times 10^{28} \mathrm{~mol} \mathrm{~s}^{-1}$ on December 22-23 (Bonev et al. 2008) and $(1.4 \pm 0.3) \times 10^{28}$ on 2008 January 3 (Barber et al. 2009).

$17 \mathrm{P}$ and $8 \mathrm{P}$ represent the most recent comets observed by Chandra. These two comets are very different in their activity and geometry. During the two Chandra observations, 17P was producing at least 100 times more water than 8P but was 2.2 times further away from the Sun. 17P was the first comet observed by Chandra at a relatively high solar latitude $(+19.1)$ while $8 \mathrm{P}$ was observed at a lower solar latitude $(+3.4)$. $8 \mathrm{P}$ shows X-ray morphology and an emission spectrum that are more typical for comets, and is also a bright comet with which to test spectral changes as a function of distance from the nucleus predicted by current solar wind charge exchange (SWCX) models (Bodewits et al. 2007). This paper focuses on the observational properties of each comet and comparison
Table 1

Observing Parameters of Comets 17P/2007 (Holmes) and 8P/2008 (Tuttle)

\begin{tabular}{lcc}
\hline \hline \multicolumn{1}{c}{ Comet Parameters } & 17P/2007 (Holmes) & 8P/2008 (Tuttle) \\
\hline Observation time (UT): & 2007 Oct 31.4 & 2008 Jan 01-04 \\
Exposure time (ks): & 30.0 & 46.8 \\
Heliocentric distance (AU): & 2.46 & 1.10 \\
Geocentric distance (AU): & 1.62 & 0.25 \\
Helio ecliptic longitude (deg): & 54 & 87.9 \\
Helio ecliptic latitude (deg): & +19 & +3.4 \\
Phase angle (deg): & 15 & 111.4 \\
\hline
\end{tabular}

to the simple SWCX model. A future paper will investigate further details of the SWCX model under a Chandra archival proposal (program CXO-09100455). The outline of this paper is the following; Section 2 presents the observations and analysis. Imaging and temporal results are presented in Sections 3 and 4, respectively. In Section 5, we discuss the total spectra, and for $8 \mathrm{P}$ we analyze spectra as a function of position from the nucleus, and spectra as a function of time. Lastly, in Section 6 we discuss the results and compare them to solar wind conditions, previous comet observations, and current SWCX models.

\section{OBSERVATIONS AND ANALYSIS}

\subsection{Observations}

Comet 17P was observed on 2007 October 31.42 for $30 \mathrm{ks}$ as part of a Director's Discretionary Time (DDT) with PI: K. Dennerl. Each pointing obtained $\sim 10 \mathrm{ks}$ on the comet for a total integration time of $30 \mathrm{ks}$. Comet $8 \mathrm{P}$ was observed as part of a cycle 9 GO program (9100452; PI: Christian) between 2008 January 1 07:31 UT and 2008 January 4 04:55 UT. Nearly 47 ks of data were obtained in three observation blocks during each of five separate pointings on 2008 January 1, 3, and 4. Details of the observations and the observing geometry are summarized in Table 1.

The Chandra observations were done in the same manner as for our previous comet observations (Lisse et al. 2001, 2005; Bodewits et al. 2007; Wolk et al. 2009), and the reader is referred to these papers for details of the observational scheme. Briefly, the ACIS-S CCD was used, providing high-resolution X-ray imaging with a plate scale of $0^{\prime \prime} .5$ pixel $^{-1}$, an instantaneous field of view (FOV) $8.3 \times 8.3$, and moderate resolution spectra $\left(\Delta E \sim 110 \mathrm{eV} \mathrm{FWHM}, \sigma_{\text {Gaussian }} \sim 50 \mathrm{eV}\right)$ in the $300-2500 \mathrm{eV}$ energy range. The observations were conducted with the comet's nucleus near the aim point in the S3 chip. No active guiding on the comet was attempted, and Chandra was able to follow the comet's motion using multiple pointings. In this method, the comet is centered in the S3 chip and allowed to drift across the chip and the Chandra pointing is updated to re-center the comet as before it moves off the chip edge.

\subsection{Analysis}

The Chandra data reduction and analysis were done in the same manner as for our previous comet observations (Lisse et al. 2001, 2005; Bodewits et al. 2007; Wolk et al. 2009), and the reader is referred to these papers for additional details. We provide only a summary of the analysis here. For each comet, combined images from each set of observations were remapped into a coordinate system moving with the comet using the "sso_freeze" algorithm, part of the Chandra Interactive Analysis of Observations (CIAO) software (Fruscione et al. 2006). Images and spectra were extracted with the CIAO tools and 


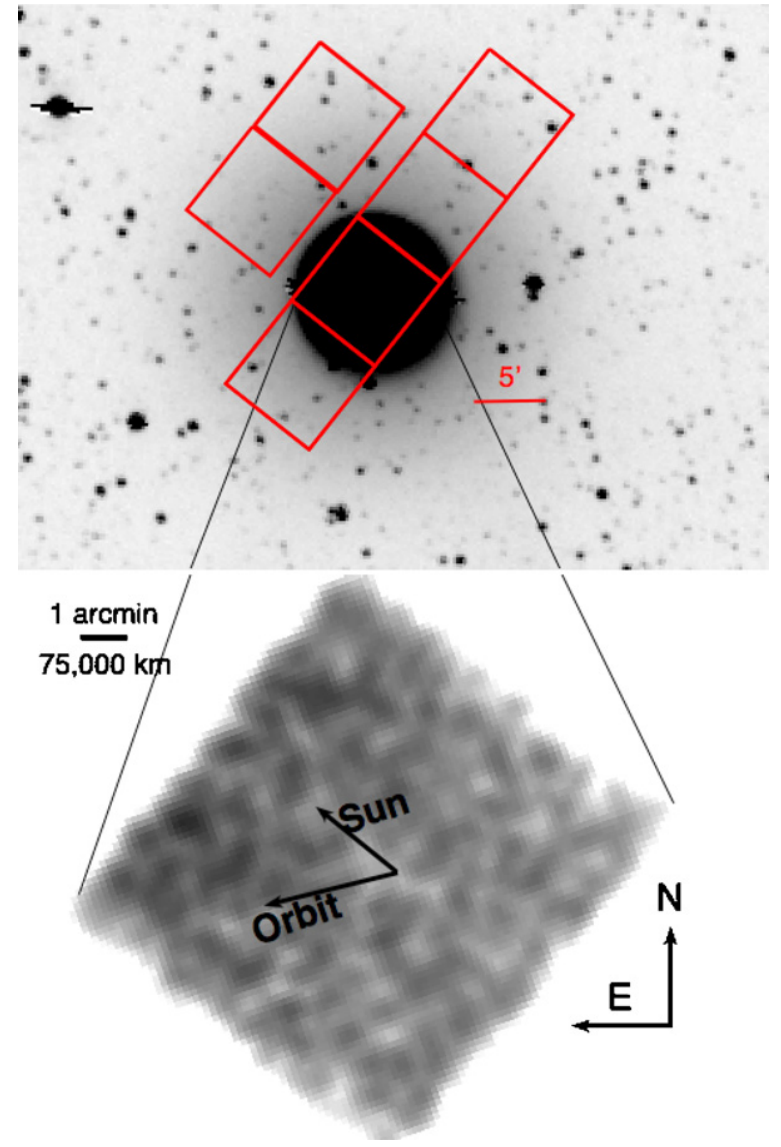

Figure 1. Chandra ACIS S3 pointing (bottom) compared to SuperWASP (top, $V$-band) observations of comet 17P/Holmes (Hsieh et al. 2007). The SuperWASP image was taken on November 1 at 04:21 UT slightly after the Chandra image of October 31 10:07 UT. The SuperWASP pixel scale is $\approx 14$ " pixel $^{-1}$.

analyzed with a combination of IDL and FTOOL packages. The X-ray spectral analysis software package XSPEC (Arnaud 1996) was used for the spectral fitting. For each comet, we extracted spectra and associated calibration products (response matrices and effective areas) using the current Chandra Interactive Analysis of Observations tools (CIAO v4).

\subsubsection{P}

The resulting effective FOV of the observations in the cometcentric coordinate system was about $9.3 \times 8.6$, and a total of approximately 7100 photons were detected in the $250-1000 \mathrm{eV}$ range (of which 5300 were between 250 and $500 \mathrm{eV}$ ) on the entire S3 chip. Thus, the average total count rate in the $250-1000 \mathrm{eV}$ energy range over the $30 \mathrm{ks}$ of observations was 0.24 counts $\mathrm{s}^{-1}$. We extracted spectra from several apertures along the top of the S3 chip and from the entire S3 chip in comet-centered coordinates. The entire S3 chip had $\sim 60 \%$ more counts than a rectangular aperture of $1100 \times 550$ pixels along the top of S3 (this region is near the CCD's readout nodes). Because of this fact, along with the large extent of the comet in the optical (much larger than Chandra's FOV), we concentrated our analysis on the spectrum extracted from the entire S3 chip. A background spectrum of the same dimensions was extracted from the bottom of the S3 chip.

The expected cometary position was verified with several other cataloged X-ray sources: 1RXS J034619.3+504139 was detected in the $\mathrm{I} 2$ chip, an un-cataloged source was found

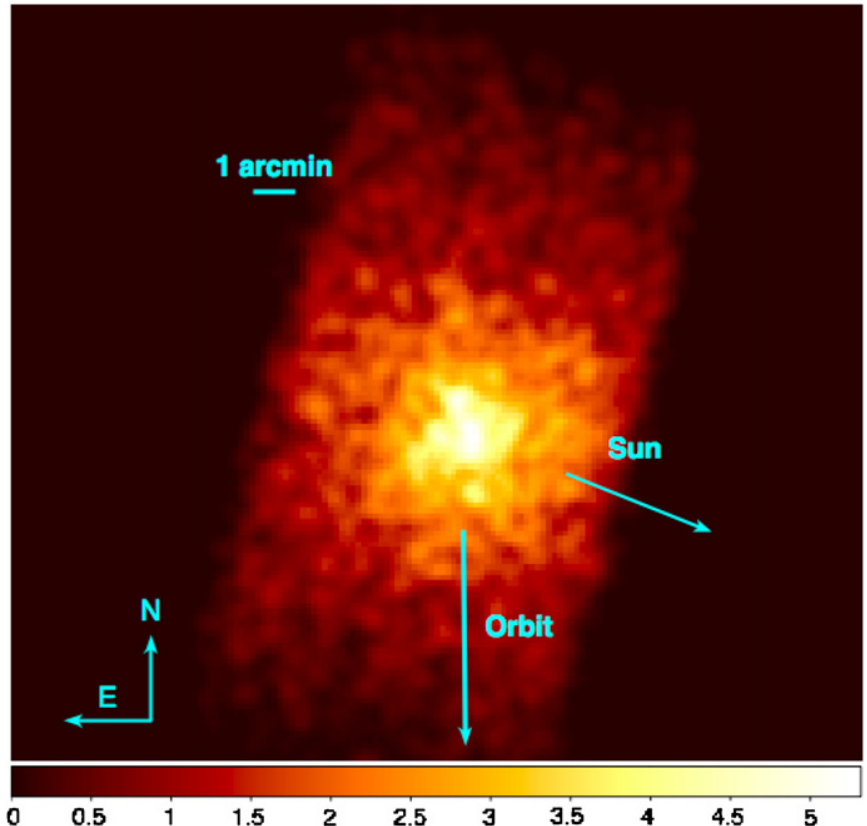

Figure 2. Chandra ACIS S3 300-1000 eV image of 8P/Tuttle. North is up and east to the left, and the position of the Sun and relative motion of the comet are indicated. The image is binned by 16 pixels and smoothed with a Gaussian filter of 3 pixels.

on the S3/S4 boundary at 03:47:17 +50:29:51, and star TYC 3338-277-1 (Voges et al. 1999) was found in the S1 chip.

Ground- and space-borne optical observations of 17P such as obtained with SuperWASP (Pollacco et al. 2006; Hsieh et al. 2007) show that around October 31 , the coma had reached a diameter larger than 45', considerably larger than the $\sim 8^{\prime} \times$ $8^{\prime}$ ACIS-S3 FOV (see Figure 1). The comet therefore extended beyond the S3 chip, but because chips I 2 and I3, and S2 and S4 are front illuminated, and have effectively no sensitivity below $\sim 500 \mathrm{eV}, 17 \mathrm{P}$ was not detected in these. Because the extent of the comet was larger than the ACIS FOV, we investigated other background spectra in addition to the spectra extracted from the lower region of the $\mathrm{S} 3$ chip. We searched the Chandra archive for ACIS-S observations at similar Galactic latitudes that would have a similar cosmic low-energy background; see Table 2. The choice of background is not trivial and has significant consequences for our analysis. It will therefore be treated in more detail in Section 5.1.

\subsection{2 $8 P$}

The effective FOV of the observations in the comet-centric coordinate system was $17^{\prime} .6 \times 99^{\prime} .2$, in which a total of approximately 9330 cometary photons detected in the $300-1000 \mathrm{eV}$ range over the entire S3 chip. Thus, the average net count rate in the $47 \mathrm{ks}$ of observations was 0.25 counts $\mathrm{s}^{-1}$. The remapped 8P comet image in the 300-1000 eV range is shown in Figure 2. With 8P's discernable image morphology, we extracted spectra from several apertures centered on the comet and from the entire S3 chip. Background spectra were also extracted from several regions away from the comet and a spectrum extracted from the top and bottom of the S3 chip was chosen as having little cometary emission. These regions are: (1) upper and lower background apertures at the top and bottom of the S3 chip, (2) a circular aperture of 100 pixels centered on the brightest central emission and labeled nucleus, (3) three concentric semicircles, noted as pandas 0,1 , and 2 centered on the brightest point of the nucleus and arranged toward the sunward side, and (4) a 
Table 2

Count Rates for 17P Using Different Background Data

\begin{tabular}{|c|c|c|c|c|c|c|c|c|}
\hline (Background) Spectrum & $\mathrm{Ra}(\mathrm{deg})$ & Decl. (deg) & $1(\mathrm{deg})$ & $b(\operatorname{deg})$ & Exposure Time (ks) & Counts $(300-1000 \mathrm{eV})$ & Counts $(300-400 \mathrm{eV})$ & 17P Flux ${ }^{\mathrm{b}} 10^{-13} \mathrm{ph} \mathrm{cm}^{-2} \mathrm{~s}^{-1}$ \\
\hline Comet 17P/2007 (Holmes) & 56.7 & +50.5 & 149.11 & -3.26 & 30 & 4577 & 2121 & 10.0 \\
\hline S3 background & & & & & 30 & 930 & 333 & 4.3 \\
\hline Obsid 4613 & 35.6 & +42.3 & 140.38 & -17.43 & 119 & 12762 & 3680 & 4.5 \\
\hline Obsid 8473 & 49.36 & +41.41 & 150.30 & -13.60 & 30 & 11250 & 2033 & 1.0 \\
\hline ACIS blank sky & $\ldots$ & $\ldots$ & $\ldots$ & $\ldots$ & 450 & 42815 & 16034 & 14.0 \\
\hline
\end{tabular}

Notes.

${ }^{\mathrm{a}}$ See the text for details

${ }^{\mathrm{b}}$ Flux for $17 \mathrm{P}$ in the $300-1000 \mathrm{eV}$ band, using this background in units of $10^{-13} \mathrm{ph} \mathrm{cm}^{-2} \mathrm{~s}^{-1}$. 


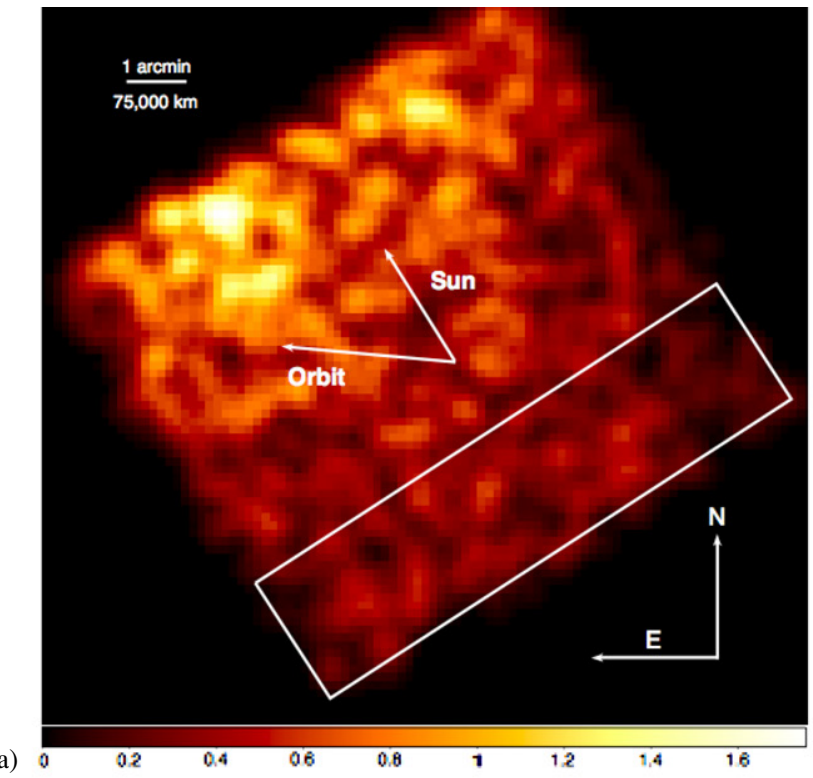

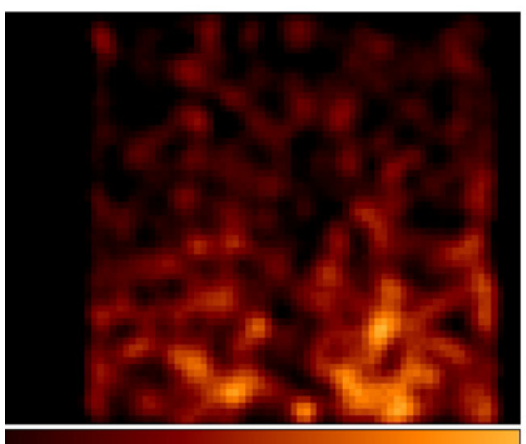

(b) $\begin{array}{llllllll}0.8 & 1 & 1.2 & 1.4 & 1.6 & 1.8 & 2 & 2.2\end{array}$

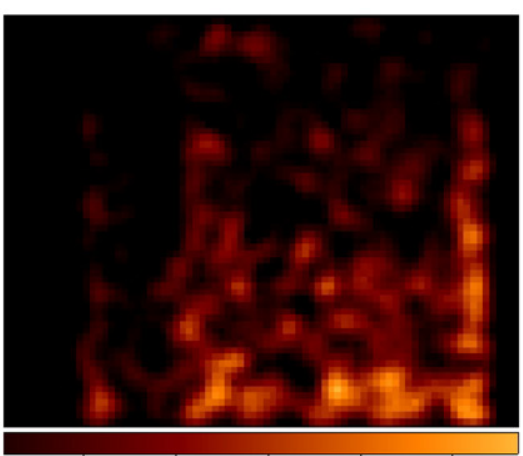

(c)

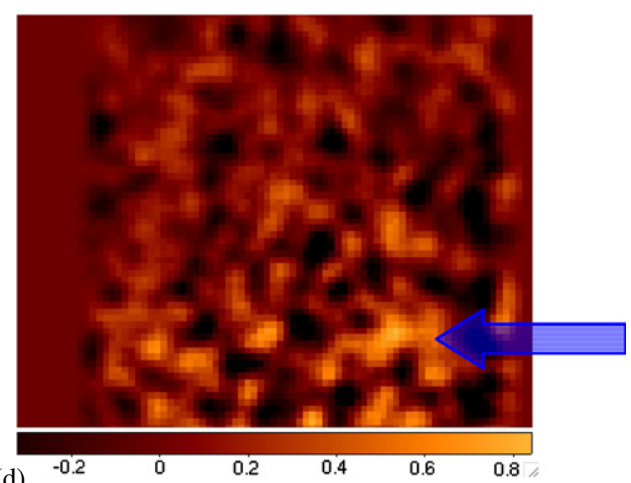

(d)

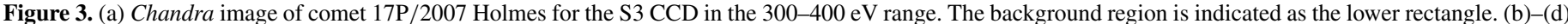

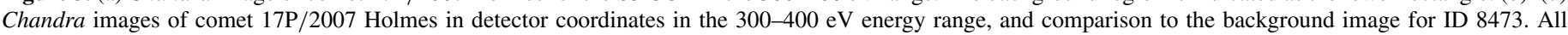

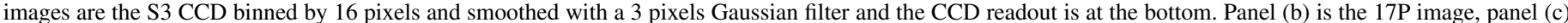

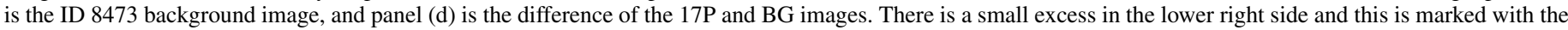
blue arrow (see the text).

semicircular region on the anti-Sun side of the nucleus labeled left panda. Net count rates for these various spectral regions are presented in Table 3. We also extracted full-chip spectra as a function of time, as the comet's X-ray emission decreased over $60 \%$ during the observation (see Section 4).

\section{IMAGING}

\section{1. $17 P$}

The reconstructed comet image is shown in Figure 1 and is compared to the wide field optical image from the SuperWASP project (Pollacco et al. 2006) in the $V$ band. Examining the ACIS S3 chip in the usual 300-1000 eV range did not show an obvious comet or a strong concentration of photons. However, the X-ray image shows the highest concentration of X-ray photons in the top part and upper left region of the $\mathrm{S} 3$ chip. This emission is more clearly visible when examining the 300-400 eV energy range, and this image is shown in Figure 3(a). The central emission does appear to have two peaks and an extension to the right rather than any recognizable morphology. These two regions have an excess of $\sim 40$ counts over background in the $300-400 \mathrm{eV}$ range, or a detection of $\sim 6 \sigma$.

We then examined several background images (Table 2, Section 5.1) in the 300-400 eV range to test if the excess soft emission could be an instrumental effect. For the ID 8473 S3 image, we also find an excess emission in the 300-400 eV range. The 17P and ID 8473 images are compared in detector coordinates in Figures 3(b)-(d). This soft excess cannot be easily distinguished from excess caused as resulting from charge transfer inefficiency of photons in the $300-400 \mathrm{eV}$ range. The charge from these photons does not propagate efficiently across the chip and is preferentially seen closer to the readout end of the chip. There may still be a small excess of soft energy photons from 17P visible in the image of 17P after background subtraction with the ID 8473 image. This image is also shown in Figure 3(d), and an excess, 40 counts (above background) in a circle with 100 pixels radius, can be seen in the lower right-hand side.

\section{2. $8 P$}

The reconstructed comet image of $8 \mathrm{P}$ is shown in Figure 2 and shows the typical crescent-shaped emission, similar to other comets, such as Hyakutake (Lisse et al. 1996), Linear S4 (Lisse et al. 2001), and C/2001 WM1 (Wegmann \& Dennerl 2005). The two-dimensional image morphology is very similar to model predictions (Wegmann et al. 2004; Wegmann \& Dennerl 2005, Bodewits et al. 2006). 8P, with its low gas production rate in 
Table 3

Observed Net Counts for 8P's Different Spectral Regions ${ }^{\mathrm{a}}$

\begin{tabular}{lcccc}
\hline \hline \multicolumn{1}{c}{ Region } & Area $\left(\mathrm{pix}^{2}\right)$ & Counts $(300-1000 \mathrm{eV})$ & Counts $(300-500 \mathrm{eV})$ & Counts $(500-700 \mathrm{eV})$ \\
\hline Full chip & $2.3 \times 10^{6}$ & 9330 & 3500 & 4040 \\
Nucleus & $3.1 \times 10^{4}$ & 530 & 220 & 260 \\
Panda 0 & $6.3 \times 10^{4}$ & 870 & 360 & 420 \\
Panda 1 & $6.3 \times 10^{4}$ & 630 & 240 & 304 \\
Panda 2 & $6.3 \times 10^{4}$ & 520 & 190 & 240 \\
Left panda & $6.3 \times 10^{4}$ & 840 & 340 & 390 \\
Background & $6.9 \times 10^{5}$ & 710 & 250 & 270 \\
\hline
\end{tabular}

Note. ${ }^{a}$ See the text for details.

the order of $2 \times 10^{28} \mathrm{~mol} \mathrm{~s}^{-1}$ (Bonev et al. 2008; Barber et al. 2009), has a morphology very similar to the low gas production rate model of $2 \mathrm{P} /$ Encke viewed at a high phase angle (e.g., see Wegmann et al. 2004, Figure 4(d)).

\section{TEMPORAL BEHAVIOR}

\section{1. $17 P$}

X-ray light curves due to charge exchange emission are a combination of the comet's gas production and the solar wind heavy ion flux. The X-ray light curve for $17 \mathrm{P}$ is constant for the entire $30 \mathrm{ks}$ observation with a net average count rate of $0.05 \pm$ 0.01 counts $\mathrm{s}^{-1}$ for our nominal background. The low count rates do not allow for a more detailed analysis of the development of the spectrum over time. Because the comet had a very large gas production rate during the observations $\left(3-5 \times 10^{29} \mathrm{~mol} \mathrm{~s}^{-1}\right)$, it must have been collisionally thick to charge exchange. The $\mathrm{X}$-ray emission then mainly comes from the outer parts of the coma, which is dominated by fragment species (rather than gases released directly from the nucleus). Short-term variations will consequently be dominated by variations in the solar wind. Based on the observed constant rate average count rate, it is to be expected that $17 \mathrm{P}$ interacted with a quiet solar wind with no large variations in its (heavy ion) composition or flux.

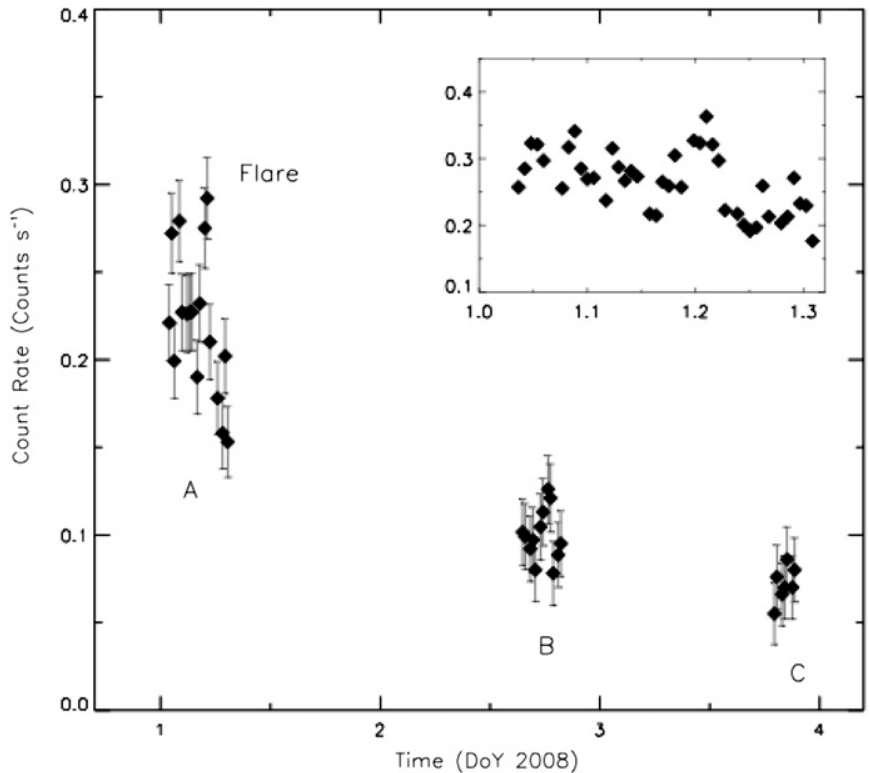

Figure 4. Chandra X-ray light curve of $8 \mathrm{P}$ shown in the $300-1000 \mathrm{eV}$ range The data are background subtracted and the bin size is $1000 \mathrm{~s}$ for the main plot. There was a modest flare observed on January 1 and this is expanded in the inset with a bin size of $500 \mathrm{~s}$. The intervals used for spectral extraction are labeled (see the text).

$$
\text { 4.2. } 8 P
$$

The 8P light curve shows a large decrease in counts between the first of the observations on January $1\left(0.4\right.$ counts $\left.\mathrm{s}^{-1}\right)$ and January $4\left(0.10\right.$ counts s $\left.^{-1}\right)$. We show the Chandra $300-1000 \mathrm{eV}$ light curve in Figure 4. A small flare was observed on January 1, but the overall trend is a decrease in count rate. $8 \mathrm{P}$ had a much smaller gas production rate than $17 \mathrm{P}$ and most of the coma will have therefore been collisionally thin to charge exchange (Bodewits et al. 2007), allowing the solar wind ions to interact with parent species close to the nucleus. X-ray brightness variations can thus indicate both variations in the gas production and in the solar wind. Short-term spectral variations however might indicate solar wind rather than cometary variations. Searches for periodic signals in the Chandra light curve indicative of the comet's rotation period (between 5.7 and $7.4 \mathrm{hr}$; Woodney et al. 2008; Harmon et al. 2008) were inconclusive as there is relatively poor phase coverage and count rates are dominated by the higher count rate data on January 1 . We investigate 8P's emission spectra in the following section.

\section{SPECTROSCOPY}

The majority of prominent X-ray emission lines can be attributed to the solar wind highly charged ions of $\mathrm{C}^{5+}, \mathrm{C}^{6+}$, $\mathrm{N}^{6+}, \mathrm{N}^{7+}$ and $\mathrm{O}^{7+}, \mathrm{O}^{8+}$, and to avoid confusion, we denote the emission line formed with a roman numeral, e.g., if an $\mathrm{O}^{7+}$ ion captures an electron it results in an $\mathrm{O}$ VII emission line near $561 \mathrm{eV}$. Spectral models for cometary X-ray emission have improved significantly since earlier simple continuum models, such as thermal bremsstrahlung fit to broadband measurements (e.g., Lisse et al. 1996). For the cometary X-ray spectra presented here we have used the SWCX model of Bodewits et al. (2007). In this SWCX model, each group of ions in a species were fixed according to their velocity-dependent emission cross sections to the ion with the highest cross section in that group. Thus, the free parameters were the relative strengths of $\mathrm{H}$ - and He-like carbon (299 and $367 \mathrm{eV}$ ), nitrogen ( $\mathrm{N} \mathrm{v}$ at 419 and $\mathrm{N}$ VI at $500 \mathrm{eV}$ ) and oxygen (O VII at 561 and $\mathrm{O}$ VIII at $653 \mathrm{eV}$ ). Additional $\mathrm{Ne}$ lines, the Ne IX forbidden, inter-combination and resonance lines around $907 \mathrm{eV}$, and the Ne x Ly-alpha at $1024 \mathrm{eV}$ were also included in our SWCX model, giving a total of eight free parameters.

\section{1. $17 P$}

The extracted S3 full-chip spectrum was very soft, with almost no emission from the O VII and O VIII features in the 500-700 eV range that are typical for comet SWCX emission. We used several different background spectra to examine 17P's $\mathrm{X}$-ray emission. First, we used a region from the lower part of the 


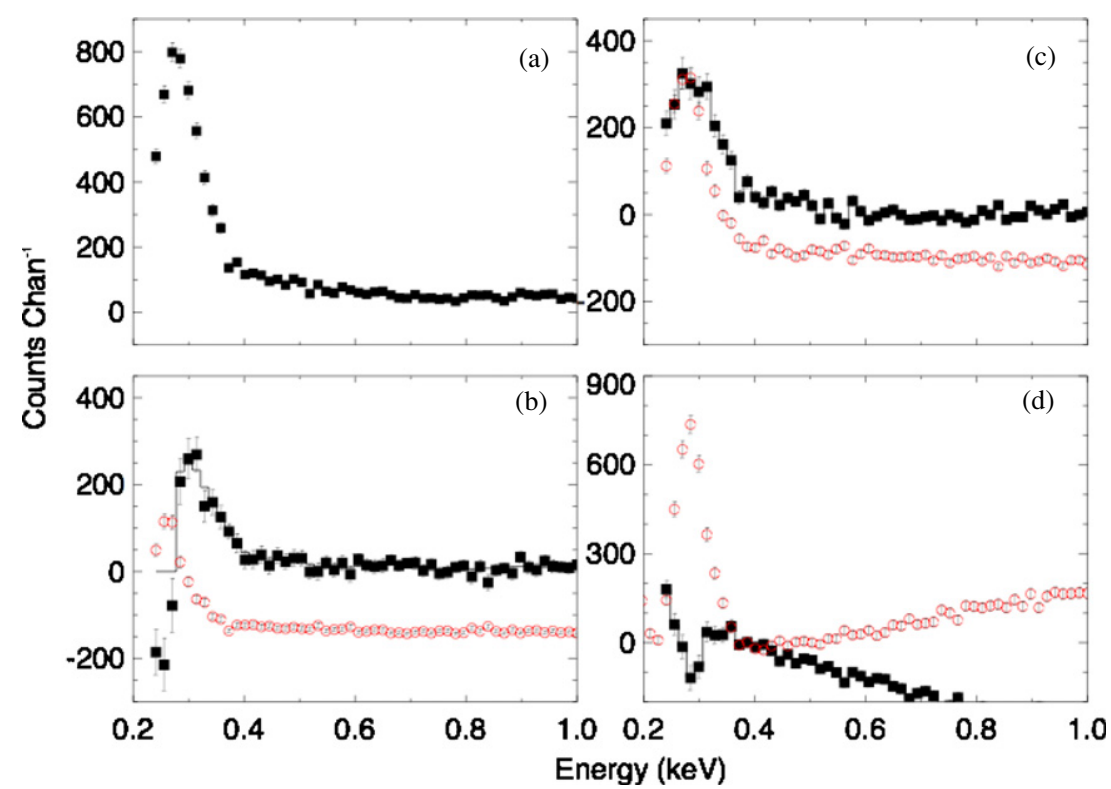

Figure 5. Comet 17P/Holmes ACIS S3 spectra. (a) S3 full-chip spectrum with no background subtracted (filled squares). (b) S3 full-chip spectrum subtracted with the S3 background spectrum extracted from lower section of the CCD. (c) The 17P full-chip spectrum subtracted with the ID 4613 background. (d) The S3 full-chip spectrum subtracted from the ID 8473 background in detector coordinates (see the text). Overplotted in panels (c) and (d) are the background spectra offset by -150 counts (open red circles).

(A color version of this figure is available in the online journal.)

S3 chip. Second, we selected a background spectrum from a 120 ks observation of SN1986J (ID 4613) which was observed in 2003 December but was the closest match to 17P's Galactic position. Third, we used a $30 \mathrm{ks}$ observation of SN2006gy (ID 8473), which also had a similar Galactic position to 17P and did not show any resolved point sources in the S3 image. And lastly, we used an ACIS 450 ks blank sky observation as a background spectrum. Other archival background spectra with shorter exposure times, and hence lower signal-to-noise ratio produced similar fluxes for the background-subtracted 17P spectrum. The $450 \mathrm{ks}$ ACIS blank sky spectrum had a very different shape and greatly over-subtracted the 500-700 eV region. We attribute this to the fact that the ACIS blank sky observations are a compilation of several different pointings and observing epochs. Thus, it is a mixture of different X-ray backgrounds with much larger O VII/VIII components and we did not perform further analysis with this background.

The total full-chip spectrum (no background removed) is shown in Figure 5(a). Using the background spectrum from the bottom of the S3 chip leaves almost no flux below $300 \mathrm{eV}$ and a soft spectrum with almost all of the emission in the $300-400 \mathrm{eV}$ range (see Figure 5(b)). Because the background region is closer to the CCD readout, the $250-300 \mathrm{eV}$ photons are preferentially higher in this region than the source region from the far side of the detector; thus the background-subtracted spectrum is negative below $300 \mathrm{eV}$.

A comparison between the total signal from the S3 full chip and the background spectrum extracted from observation ID 4613 (after removal of point sources) is shown in Figure 5(c). This background spectrum had a $200-400 \mathrm{eV}$ flux similar to that attributed to $17 \mathrm{P}$ and a similar lack of emission at energies greater than $500 \mathrm{eV}$.

Lastly, we also used a background extracted from the entire S3 chip of the SN2006gy (ID 8473) observation in detector coordinates. This spectrum has a very similar $200-400 \mathrm{eV}$ flux to $17 \mathrm{P}$, but has a much larger excess at energies greater than $500 \mathrm{eV}$, probably from the soft X-ray background (see Figure 5(d)). Thus the background-subtracted 17P spectrum is negative for fluxes above approximately $450 \mathrm{eV}$ and only shows a small positive excess in the $250-400 \mathrm{eV}$ range.

We then fit the 17P spectrum with each of the three backgrounds using the SWCX model of Bodewits et al. (2007). The spectral parameters are given in Table 4 and the spectrum with model components is shown in Figure 6. We find a flux of $4.3 \pm 0.7 \times 10^{-13} \mathrm{erg} \mathrm{cm}^{-2} \mathrm{~s}^{-1}$ in the $300-1000 \mathrm{eV}$ range using the 17P S3 lower background, a flux of $4.5 \pm 0.7 \times$ $10^{-13} \mathrm{erg} \mathrm{cm}^{-2} \mathrm{~s}^{-1}$ for the ID 4613 background, and a flux of $0.5 \pm 0.3 \times 10^{-13} \mathrm{erg} \mathrm{cm}^{-2} \mathrm{~s}^{-1}$ for the ID 8473 background. We note that over $90 \%$ of the observed flux is in the $300-500 \mathrm{eV}$ range, but give the $300-1000 \mathrm{eV}$ flux for comparison to pervious studies (e.g., Bodewits et al. 2007).

We also tested the effect of increasing the background on 17P's spectrum. Increasing the background about $50 \%$ removed all comet flux above about $400 \mathrm{eV}$ and the $300-400 \mathrm{eV}$ spectrum can be fit with only two emission lines, C v at $299 \mathrm{eV}$ and C vi at $367 \mathrm{eV}$ giving a reduced $\chi^{2}$ of 0.7 . The derived flux for this increased background was $1.6 \pm 0.3 \times 10^{-13} \mathrm{erg} \mathrm{cm}^{-2} \mathrm{~s}^{-1}$, and thus it has two-third less flux than the nominal background subtraction. Clearly, we are over-subtracting the background with this scaling, but it does give us an additional lower limit to the flux.

\section{2. $8 P$}

The 8P spectrum shows the typical strong $\mathrm{O}$ VII and moderate $\mathrm{Cv}, \mathrm{C}$ vi, Nvi, NviI, NeIx, and Nex emission. The fitted SWCX model had a reduced $\chi^{2}=1.2$ and is a reasonably good fit for 47 degrees of freedom. These spectral parameters are given in Table 5 and the 8P full-chip spectrum is shown in Figure 7. We find a flux of $9.4 \pm 0.8 \times 10^{-13} \mathrm{erg} \mathrm{cm}^{-2} \mathrm{~s}^{-1}$ in the $300-1000 \mathrm{eV}$ range. This gives an X-ray luminosity of $1.7 \pm 0.1 \times 10^{14} \mathrm{erg} \mathrm{s}^{-1}$ in this energy range. 

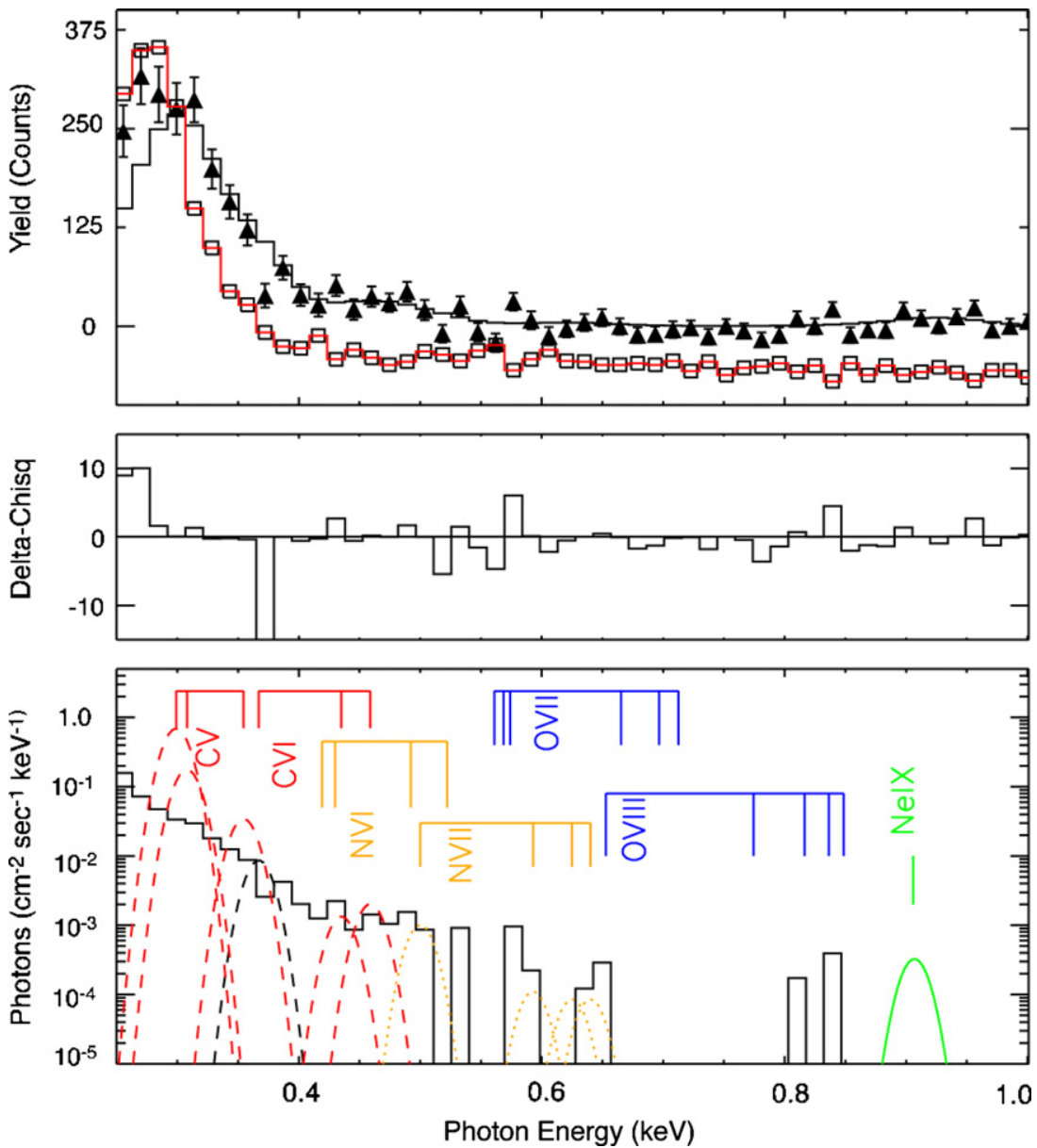

Figure 6. Details of the SWCX fit for the spectrum of comet 17P/Holmes. Top panel: background subtracted S3 full-chip spectrum (filled triangles). The background (open squares, red line) used is the ID 4613 background (see the text), scaled with a factor of 0.25 , and offset downward by 100 counts for ease of presentation. Middle panel: residuals of the SWCX fit. Bottom panel: SWCX $700 \mathrm{~km} \mathrm{~s}^{-1}$ model and observed spectrum indicating the different lines and their strengths. The unfolded model is scaled above the emission for the ease of presentation.

(A color version of this figure is available in the online journal.)

Table 4

Results of the Spectral Fit to the 17P/Holmes Observations, with Different Backgrounds ${ }^{\mathrm{a}}$

\begin{tabular}{lccccc}
\hline \hline$E_{\text {line }}(\mathrm{eV})$ & Ion Line & $\begin{array}{c}\text { Flux }^{\mathrm{b}} \\
\text { 17P S3 BG }\end{array}$ & $\begin{array}{c}\text { Flux }^{\mathrm{b}} \\
\text { ID 4613 BG }\end{array}$ & $\begin{array}{c}\text { Flux }^{\mathrm{b}, \mathrm{c}} \\
\text { ID 4613 }\end{array}$ & $\begin{array}{c}\text { Flux }^{\mathrm{b}} \\
\text { ID 8473 }\end{array}$ \\
\hline 299 & C v f+r+i & $930 \pm 120$ & $1100 \pm 100$ & $480 \pm 130$ & $160 \pm 100$ \\
367.5 & C vi Ly-a & $10.5 \pm 4.0$ & $11.5 \pm 5.0$ & $<4.0$ & $<5.0$ \\
419.8 & N vi f+r+i & $<7.0$ & $<6.0$ & $\ldots$ & $\ldots$ \\
500.3 & N viI Ly-a & $1.9 \pm 1.5$ & $2.7 \pm 0.9$ & $\ldots$ & $\ldots$ \\
561.1 & O vII f+r+i & $<1.3$ & $<1.5$ & $\ldots$ & $\ldots$ \\
653.5 & O viII Ly-a & $<1.0$ & $<0.9$ & $\ldots$ & $\ldots$ \\
907 & Ne IX f+r+i & $<0.8$ & $<0.8$ & $\ldots$ & $\ldots$ \\
1024 & Ne x & $<0.9$ & $<0.8$ & 0.7 & 1.2 \\
$\chi 2 /$ dof & & 0.6 & 1.1 & 1.6 & 0.5 \\
Total flux & $\left(10^{-13} \mathrm{erg} \mathrm{cm}^{-2} \mathrm{~s}^{-1}\right)$ & 4.3 & 4.5 & & $\ldots$ \\
\hline
\end{tabular}

Notes.

${ }^{\text {a }}$ See the text for details.

${ }^{\mathrm{b}}$ Line fluxes in units of $10^{-5} \mathrm{ph} \mathrm{cm}^{-2} \mathrm{~s}^{-1}$.

${ }^{\mathrm{c}}$ Background multiplied by a factor of 1.5 .

Spectra extracted as a function of distance from the comet's nucleus and as a function of time during the observation showed remarkable changes in the carbon and oxygen emission. Spectra at distances closer to the comet's nucleus showed a decrease in the O vIII flux consistent with SWCX models. The spectral fits to these regions are summarized in Table 5, and the spectra are shown in Figure 8. Spectra extracted as a function of time showed a dramatic change from the brightest interval (A) on January 1 to the faintest interval on January 4. On January 1, the oxygen $(\mathrm{O}$ VII $+\mathrm{O}$ VIII) emission is approximately two times stronger than the combined low-energy $300-500 \mathrm{eV}$ emission during the highest count rates and decreases to about $50 \%$ 


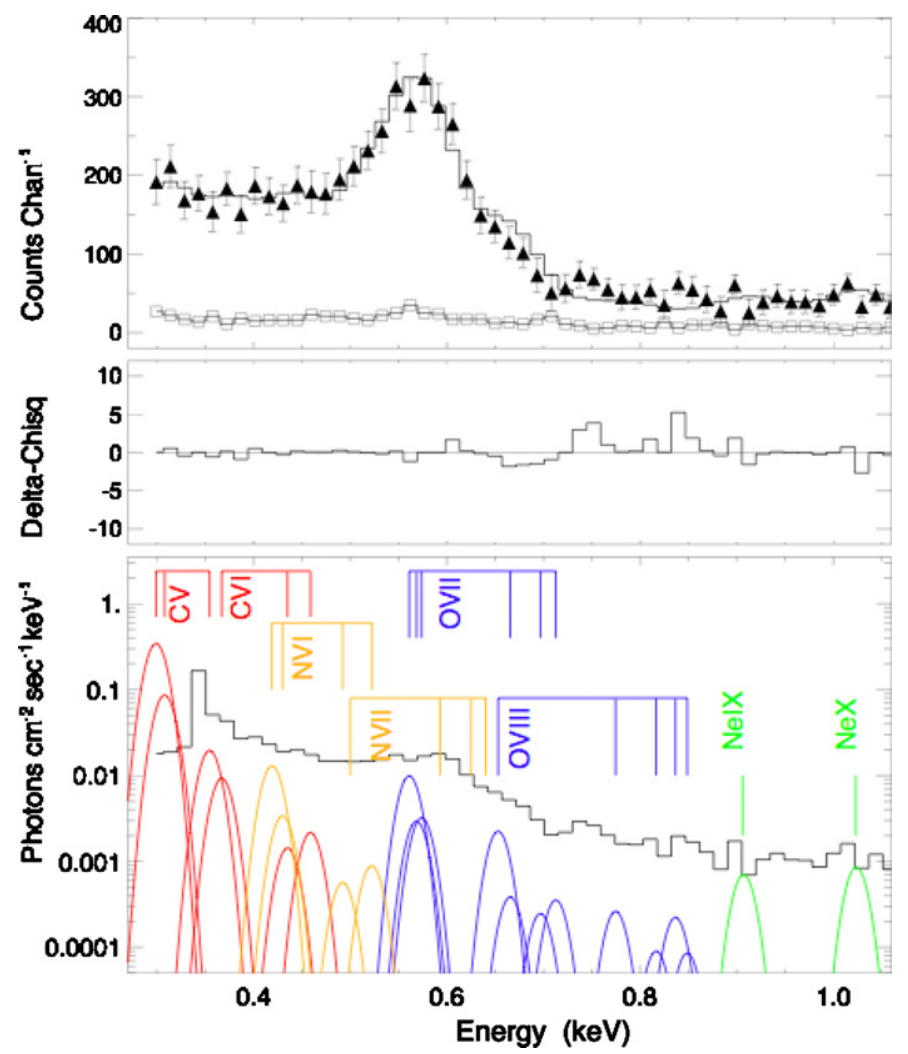

Figure 7. Details of the SWCX fit for the spectrum of comet 8P. Top panel: background subtracted S3 full-chip spectrum (filled triangles). The background (open squares) used. Middle panel: residuals of the SWCX fit in units of $\chi^{2}$. Bottom panel: SWCX $400 \mathrm{~km} \mathrm{~s}^{-1}$ model and observed spectrum indicating the different lines and their strengths. The unfolded model is scaled above the emission for the ease of presentation.

(A color version of this figure is available in the online journal.)

greater on January 2 , to being about equal on January 4 . The January 4 spectrum has very weak overall emission and is approximately $60 \%$ fainter than the January first emission. The small flare spectrum on January 1 has a very similar shape to the overall spectrum on that day, but about $15 \%$ more flux. The spectral fits to these regions are also summarized in Table 6, and the spectra are shown in Figure 9. We compare these trends in both the distance from the nucleus and time to the current SWCX models in Section 6.

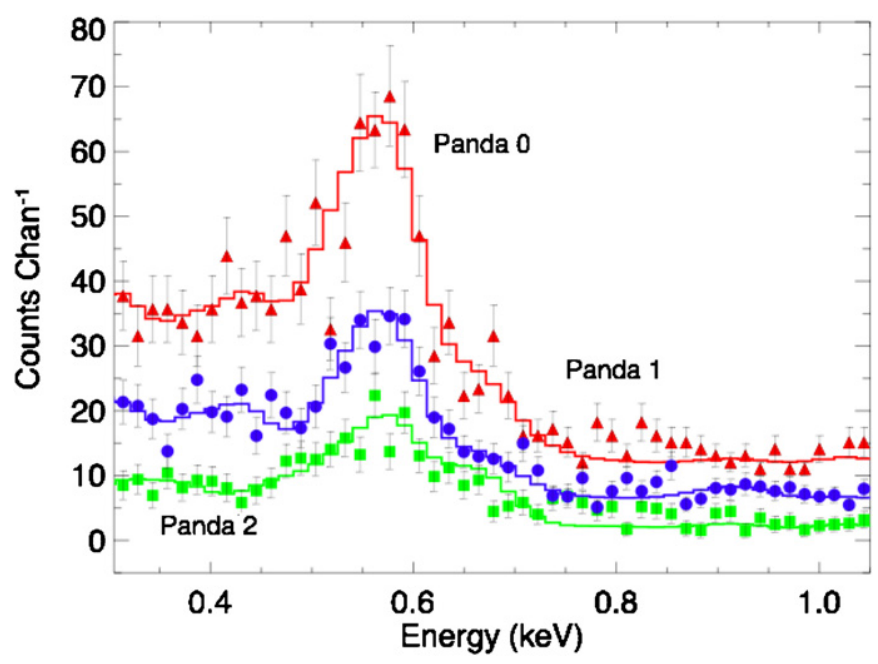

Figure 8. Comparison of spectra for the larger-scale structure of comet $8 \mathrm{P}$ for the entire observation. We compare three annular apertures (pandas) centered on the nominal nucleus (see Section 2.2). Spectra are fit with the SWCX model with a solar wind velocity of $400 \mathrm{~km} \mathrm{~s}^{-1}$. Pandas 1 (blue) and 2 (green) are scaled by their areas relative to panda 0 , and panda 0 (red) and panda 1 are offset above panda 2, by 5 and 10 counts per channel, respectively, for ease of presentation. All spectra show relatively strong emission from $\mathrm{O}$ VII, and the innermost region (panda 2, green) shows the strongest $\mathrm{O}$ VIII emission (see the text).

(A color version of this figure is available in the online journal.)

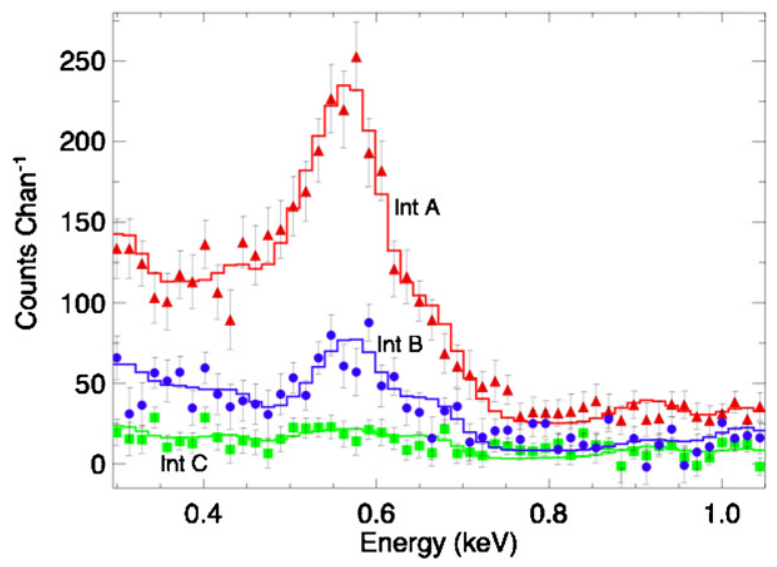

Figure 9. Comparison of $8 \mathrm{P}$ full-chip spectra as a function of time. Spectra are fit with the same SWCX model as Figure 8, and shown are intervals A, B, and $\mathrm{C}$ as identified in Section 2.2. The spectrum of the small flare is not shown, but has a very similar shape as interval $\mathrm{A}$, and has $\sim 15 \%$ more flux (see the text).

(A color version of this figure is available in the online journal.)

Table 5

Results of the Spectral Fit to the 8P/Tuttle Observations for Different Extraction Regions

\begin{tabular}{|c|c|c|c|c|c|c|c|}
\hline$E_{\text {line }}(\mathrm{eV})$ & Line $^{a}$ & Full Chip & Panda 0 & Panda 1 & Panda 2 & Left Panda & Nucleus \\
\hline 299 & $\mathrm{Cvf}+\mathrm{r}+\mathrm{i}$ & $4570 \pm 700$ & $442 \pm 100$ & $686 \pm 101$ & $482 \pm 133$ & $376 \pm 120$ & $197 \pm 92$ \\
\hline 367.5 & C vi Ly-a & $209 \pm 90$ & $23 \pm 16$ & $<16$ & $41 \pm 16$ & $37 \pm 15$ & $25 \pm 13$ \\
\hline 419.8 & $\mathrm{~N}$ VI $\mathrm{f}+\mathrm{r}+\mathrm{i}$ & $128 \pm 44$ & $17 \pm 8$ & $29 \pm 7$ & $<10$ & $<9$ & $<6$ \\
\hline 500.3 & N vil Ly-a & $53 \pm 16$ & $7 \pm 3$ & $4 \pm 3$ & $8 \pm 3$ & $9 \pm 3$ & $5 \pm 3$ \\
\hline 561.1 & $\mathrm{O}$ VII $\mathrm{f}+\mathrm{r}+\mathrm{i}$ & $165 \pm 11$ & $21 \pm 2$ & $26 \pm 3$ & $22 \pm 2$ & $18 \pm 2$ & $13 \pm 3$ \\
\hline 653.5 & O vIII Ly-a & $59 \pm 6$ & $4 \pm 1$ & $5 \pm 1$ & $11 \pm 1$ & $4 \pm 1$ & $2 \pm 1$ \\
\hline 907 & NeIX $f+r+i$ & $11 \pm 2$ & $<0.2$ & $1.1 \pm 0.2$ & $1.3 \pm 0.3$ & $0.3 \pm 0.2$ & $<0.1$ \\
\hline 1024 & $\mathrm{Nex}$ & $15 \pm 2$ & $0.5 \pm 0.2$ & $0.8 \pm 0.2$ & $1.8 \pm 0.3$ & $0.4 \pm 0.2$ & $0.3 \pm 0.2$ \\
\hline$\chi 2 /$ dof & & 1.2 & 1.3 & 0.9 & 1.3 & 1.1 & 1.0 \\
\hline Total flux ${ }^{b}$ & $\left(10^{-13} \mathrm{erg} \mathrm{cm}^{-2} \mathrm{~s}^{-1}\right)$ & 9.36 & 1.00 & 1.17 & 1.18 & 0.96 & 0.61 \\
\hline
\end{tabular}

Notes.

${ }^{\text {a }}$ Line fluxes in units of $10^{-6} \mathrm{ph} \mathrm{cm}^{-2} \mathrm{~s}^{-1}$.

${ }^{\mathrm{b}}$ Flux for $8 \mathrm{P}$ in units of $10^{-13} \mathrm{erg} \mathrm{cm}^{-2} \mathrm{~s}^{-1}$ in the $0.3-1.0 \mathrm{keV}$ band. 
Table 6

Results of the Spectral Fit to 8P/Tuttle Observations ("Full Chip"), Extracted at Different Time Intervals

\begin{tabular}{|c|c|c|c|c|c|}
\hline \multirow{2}{*}{$\begin{array}{l}E_{\text {line }} \\
(\mathrm{eV})\end{array}$} & \multirow[t]{2}{*}{ Line $^{a}$} & \multicolumn{3}{|c|}{ Interval } & \multirow[t]{2}{*}{ Flare } \\
\hline & & A & $\mathrm{B}$ & $\mathrm{C}$ & \\
\hline 299 & $\mathrm{C} v \mathrm{f}+\mathrm{r}+\mathrm{i}$ & $6480 \pm 748$ & $4483 \pm 710$ & $2847 \pm 538$ & $7572 \pm 1257$ \\
\hline 367.5 & C VI Ly-a & $120 \pm 110$ & $100 \pm 94$ & $<50$ & $<220$ \\
\hline 419.8 & $\mathrm{~N}$ VI $\mathrm{f}+\mathrm{r}+\mathrm{i}$ & $187 \pm 55$ & $120 \pm 52$ & $89 \pm 33$ & $227 \pm 84$ \\
\hline 500.3 & N vII Ly-a & $95 \pm 19$ & $20 \pm 16$ & $32 \pm 18$ & $120 \pm 37$ \\
\hline 561.1 & $\mathrm{O}$ vII $\mathrm{f}+\mathrm{r}+\mathrm{i}$ & $208 \pm 20$ & $104 \pm 15$ & $41 \pm 12$ & $251 \pm 30$ \\
\hline 653.5 & O viII Ly-a & $61 \pm 7$ & $53 \pm 7$ & $35 \pm 8$ & $71 \pm 12$ \\
\hline 907 & NeIX $f+r+i$ & $10 \pm 2$ & $12 \pm 2$ & $9 \pm 2$ & $11 \pm 3$ \\
\hline 1024 & $\mathrm{Ne} x$ & $13 \pm 2$ & $14 \pm 3$ & $12 \pm 3$ & $10 \pm 3$ \\
\hline$\chi^{2 / \text { dof }}$ & & 0.87 & 1.42 & 1.01 & 0.60 \\
\hline Total flux ${ }^{\mathrm{b}}$ & $\left(10^{-13} \mathrm{erg} \mathrm{cm}^{-2} \mathrm{~s}^{-1}\right)$ & 10.7 & 6.5 & 3.7 & 12.3 \\
\hline
\end{tabular}

Notes.

${ }^{\text {a }}$ Line fluxes in units of $10^{-6} \mathrm{ph} \mathrm{cm}^{-2} \mathrm{~s}^{-1}$.

${ }^{\mathrm{b}}$ Flux for $8 \mathrm{P}$ in units of $10^{-13} \mathrm{erg} \mathrm{cm}^{-2} \mathrm{~s}^{-1}$ in the $0.3-1.0 \mathrm{keV}$ band.

\section{DISCUSSION}

\subsection{Did Chandra Detect 17P?}

In the preceding sections, we described how we searched for signatures of X-rays from comet $17 \mathrm{P}$ by examining both the images and spectra. Using a suitable background from the lower part of the S3 chip or from a similar Galactic position (ID 4613) we derive a flux of $4.5 \pm 0.7 \times 10^{-13} \mathrm{erg} \mathrm{cm}^{-2} \mathrm{~s}^{-1}$. Much of this emission may be the result of charge transfer inefficiency, which artificially enhances emission in the $200-400 \mathrm{eV}$ range from the side of the detector furthest from the readout amplifier. Subtracting a spectrum in detector coordinates from ID 8473 gives a flux of only $0.5 \pm 0.3 \times 10^{-13} \mathrm{erg} \mathrm{cm}^{-2} \mathrm{~s}^{-1}$, and this may be the true emission from the comet. These fluxes give a range of luminosities for $17 \mathrm{P}$ of $0.36-3.3 \times 10^{15} \mathrm{erg} \mathrm{s}^{-1}$. Neither method yielded conclusive evidence of cometary X-rays from 17P during our 30 ks Chandra observation.

One possible explanation for the lack of image morphology could be the large extent of the coma due to the comet's high gas production rate and its large heliocentric distance. If the coma were collisionally thick to charge exchange outside the S3 detector's $8.3 \times 8.3 \mathrm{FOV}$, this could explain the extremely soft X-ray spectrum as charge exchange reactions decrease the average charge of solar wind ions along their trajectory through the gas around the comet, and will produce softer X-ray emission in subsequent reactions. At the distance of 17P, 8.3 corresponds to approximately $5.8 \times 10^{5} \mathrm{~km}$. According to the comet-solar wind models that we discussed in our survey paper (Bodewits et al. 2007), it is most unlikely that there is a notable collisional opacity effect on the ionic flux ratios at these distances from the nucleus, even for large gas production rates (see, e.g., Figure 7 in Bodewits et al. 2007).

We note that Chandra ACIS-S is not very well suited for the study of these soft energies. If we believe we did detect 17P near the outer edge of the S3 chip we then have to explain how the $\mathrm{X}$-rays can originate $\sim 3.5$ or $\sim 175,000 \mathrm{~km}$ from the nominal nucleus. The brightest portion of the emission in the re-mapped coordinates is in the comet-Sun direction. Optical studies of $17 \mathrm{P}$ suggest a spherically symmetric gas distribution, in which case the X-ray emission would be concentrated on the sunward side of the coma along the comet-Sun axis. Uncertainties from outgassing or other dynamical effects may therefore be needed to explain why the comet's X-rays are at a large distance from the nominal nucleus, but there is a significant detection above background in the 300-400 eV energy range.

Due to the high latitude and large geocentric distance of 17P, it is unreasonable to assume solar wind conditions measured by any of the spacecraft located at L1. The Ulysses spacecraft was positioned at a higher latitude and larger heliocentric distance. Around our observations, it measured fast coronal hole wind, but the variations do not allow for a simple extrapolation to the position of the comet.

\subsection{Spectral Changes of $8 P$}

Spectra extracted as a function of distance from the nucleus do show an interesting trend that the ratios of $\mathrm{C} \mathrm{VI} / \mathrm{C} \mathrm{V}, \mathrm{O}$ VIII/O VII decrease when comparing outer regions of the coma to inner regions. These ratios are shown in Figure 10(a). These observed changes are expected in the SWCX model as solar wind ions lose their charge capturing electrons from the cometary neutrals (Bodewits et al. 2007). The $\mathrm{C} \mathrm{VI} / \mathrm{C}$ v and $\mathrm{N}$ vII/N vi ratios also decrease about a factor of 2 from the outer to inner regions, although no strong difference is observed between the two inner regions (panda 0 and panda 1 ).

Spectra extracted as a function of time, as $8 \mathrm{P}$ showed a $60 \%$ decrease in count rate, showed that the spectrum flattened and the usual ratio of oxygen emission being 50\% larger than the $\mathrm{C}+\mathrm{N}$ changes to be nearly equal for the lowest count rates. Similarly, the ratios of O VIII/O VII became more equal from there values of 0.3 at the start of the observations. These ratios for the spectra extracted as a function of time are shown in Figure 10(b). Thus, $\mathrm{Cv}$ and $\mathrm{O}$ VII emission dominates 8P's spectrum during the brightest and most flare-like intervals.

To compare our observations with solar wind conditions measured around L1, we applied a simple solar wind extrapolation scheme, outlined and discussed in our survey paper (Bodewits et al. 2007). In brief, one assumes constant outflow and tangential velocities and calculates the time lag or leads between spacecraft and comet to get an idea about the solar wind conditions resulting in the emitted X-rays. This extrapolation is a strong simplification of solar wind progression, and is only of use in relatively stable solar wind conditions, and when the comet is close to Earth. Further details of this procedure are also outlined in Neugebauer et al. (2000). We obtained estimates of the solar wind conditions at comet 8P during the Chandra observations from Solar and Heliospheric Observatory $(\mathrm{SOHO})$ 

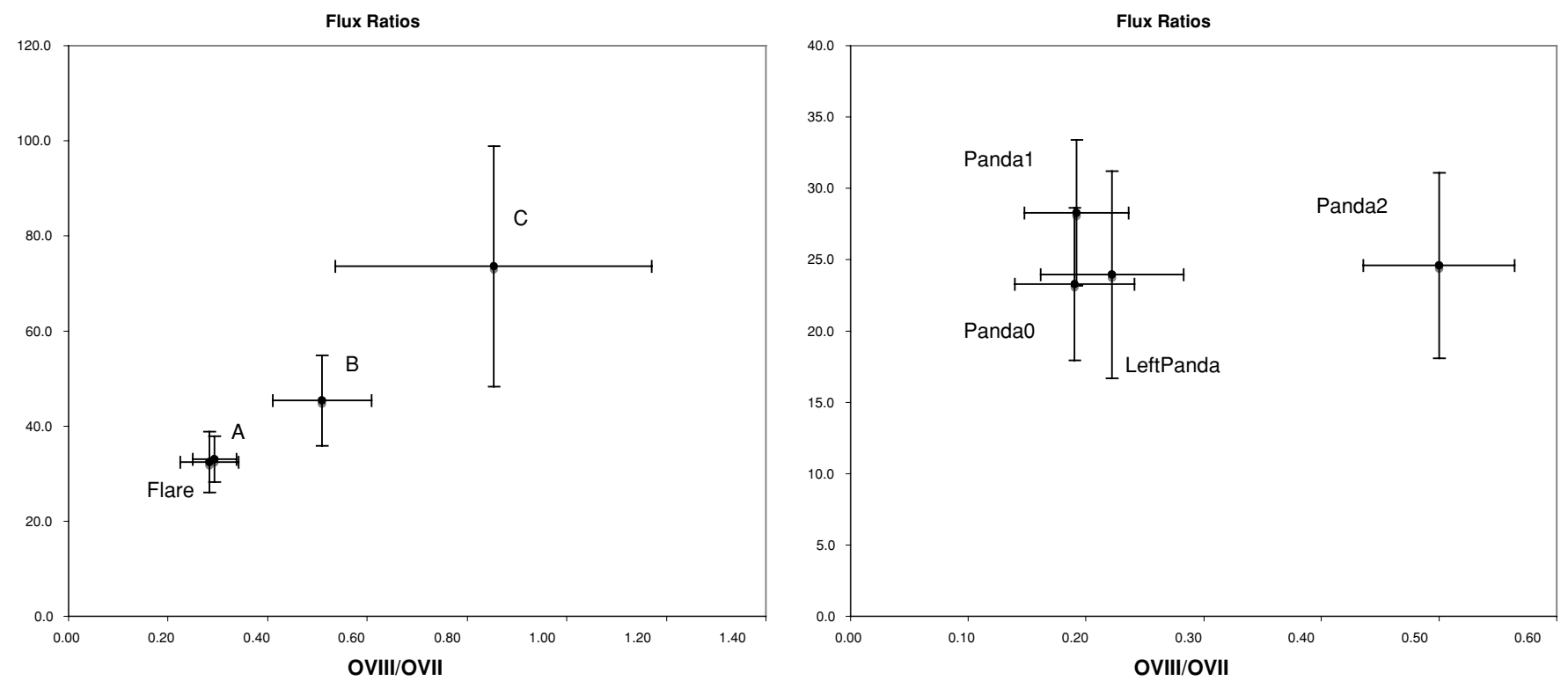

Figure 10. Emission-line flux ratios from spectral fitting. Both figures show the ratio of the combined carbon plus nitrogen emission to that of the $\mathrm{O}$ vII emission plotted against the $\mathrm{O}$ VIII(653)/O VII(561) ratio. A. The left-hand figure shows these ratios for spectra extracted as a function of time. Each interval is labeled (A, B, C \& Flare). Interval $\mathrm{A}$ and the flare spectra show the largest $\mathrm{O}$ VII emission and the lowest $\mathrm{O}$ VIII/O vII ratios. Interval $\mathrm{C}$, the lowest flux interval, shows similar amounts of VII and $\mathrm{O}$ VIII and their ratio is nearly unity. B. The right-hand figure shows ratios from the spectral regions panda $0,1,2$ and the region behind the nucleus, noted as left panda. Here the region further from the nucleus shows the largest O VIII emission as expected from the simple SWCX model, but the combined carbon + nitrogen emission compared to the O VII emission stays nearly constant (see the text).
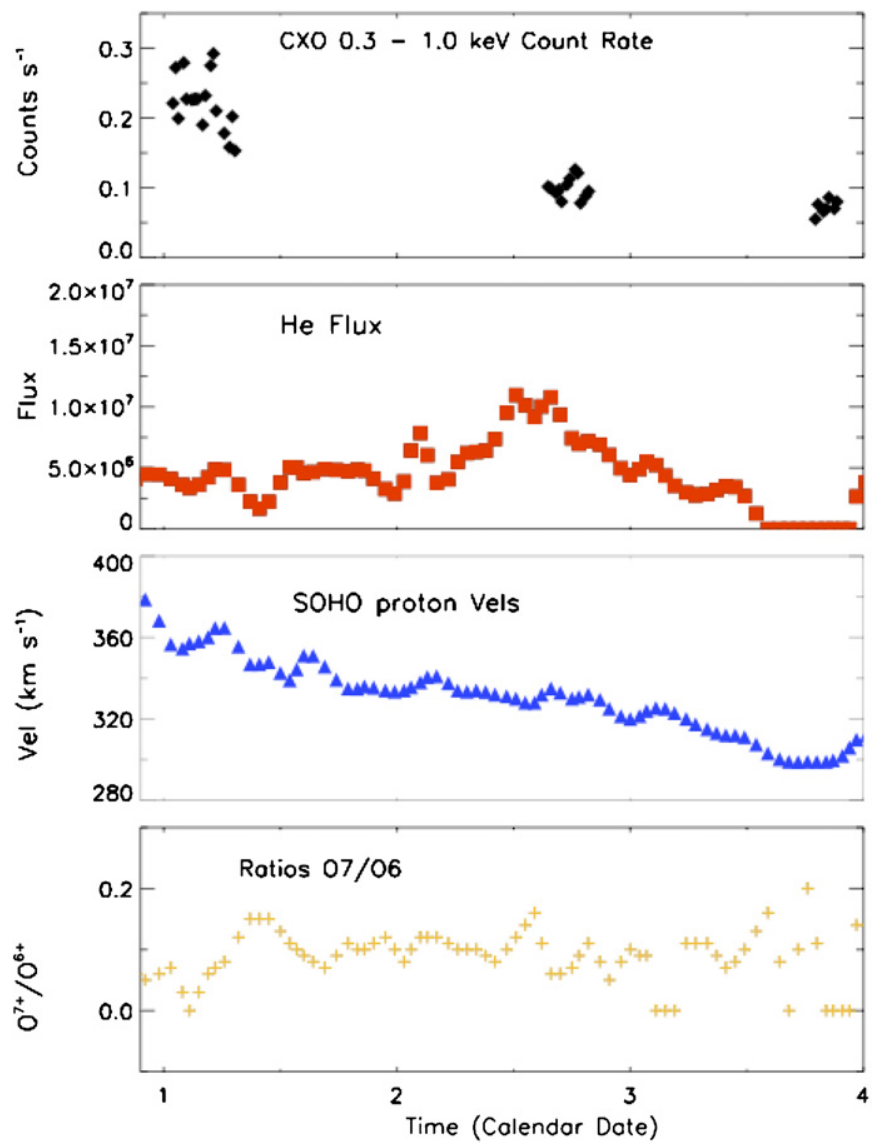

Figure 11. Chandra $8 \mathrm{P}$ light curve and measured solar wind parameters from ACE/SWICS and SOHO/PM. All data are mapped from the position of the spacecraft to that of the comet using a simple extrapolation for the solar wind (see Section 6.2). (a) The top panel shows the Chandra X-ray light curve. Panel (b) shows the $\mathrm{He}^{2+}$ flux and panel (c) the proton solar wind velocities from SOHO. The lowest panel, panel (D), shows the $\mathrm{O}^{7+} / \mathrm{O}^{6+}$ (yellow points).

(A color version of this figure is available in the online journal.) and Advanced Composition Explorer (ACE) spacecraft, shown in Figure 11. From these solar wind data, we observe that 8P interacted with a quiet, slow solar wind moving at approximately $300-400 \mathrm{~km} \mathrm{~s}^{-1}$. The solar wind on January 1 reached nearly $400 \mathrm{~km} \mathrm{~s}^{-1}$ as observed in the $A C E$ ion and $S O H O$ proton velocities data. The wind velocity decreased to below $300 \mathrm{~km} \mathrm{~s}^{-1}$ on January 4 , and the decrease in the Chandra count rate reflects this. We compare the $8 \mathrm{P}$ X-ray light curve and the solar wind data in Figure 11 . The $\mathrm{O}^{7+} / \mathrm{O}^{6+}$ ion data show minimal variation and are always below 0.2 during the observation, indicating a steady solar wind with a constant freeze-in temperature, but do show a slight increase near the observed flare on January 1.

\section{3. $17 P$ and $8 P$ and Other Comets}

We derived a range of luminosities for $17 \mathrm{P}$ of $0.36-3.3 \times$ $10^{15} \mathrm{erg} \mathrm{s}^{-1}$, and found 8P's luminosity to be $1.7 \times 10^{14} \mathrm{erg} \mathrm{s}^{-1}$. Although our nominal luminosity of $17 \mathrm{P}\left(3.3 \times 10^{15} \mathrm{erg} \mathrm{s}^{-1}\right)$ is on the lower end for X-ray luminosities of comets (see Figure 12 in Wolk et al. 2009), it is concentrated entirely in the 300-400 eV range. $8 \mathrm{P}$ was a relatively faint $\mathrm{X}$-ray comet and only $2 \mathrm{P} /$ Encke and 73P/Schwassmann-Wachmann 3's fragment B were less luminous in X-rays than 8P. These three comets all had comparable gas production rates $\left(\sim 10^{28} \mathrm{~mol} \mathrm{~s}^{-1}\right)$ and were observed during moderate solar wind conditions. If not for the solar outburst on January 1, 8P would have been even three times fainter.

Both comets encountered unique solar wind conditions. Solar wind condition for $8 \mathrm{P}$ and $17 \mathrm{P}$ and a comparison to several of the comets from the previous Chandra survey (Bodewits et al. 2007) are given in Table 7. We include comet C/1999 S4 (Linear S4, LS4), which encountered a $\sim 600 \mathrm{~km} \mathrm{~s}^{-1}$ wind and has been a well-studied comet to use for comparison. We also included comet C/1999 T1 (Mcaught-Hartley, hereafter McH), which also encountered a solar wind designated as having a "hot" composition and with a bulk velocity of $353 \mathrm{~km} \mathrm{~s}^{-1}$ (Bodewits et al. 2007). Previously, we had derived solar wind 
Table 7

Solar Wind Conditions and Selected Parameters for 17P and $8 \mathrm{P}$ and Comparison to Comets With Different Solar Wind Conditions from Bodewits et al. (2007)

\begin{tabular}{lcccc}
\hline \hline \multirow{1}{*}{ Comet } & Q gas & Type & \multicolumn{2}{c}{ Solar Wind } \\
\cline { 4 - 5 } & $\left(10^{28} \mathrm{~mol} \mathrm{~s}^{-1}\right)$ & & $V_{\mathrm{p}}\left(\mathrm{km} \mathrm{s}^{-1}\right)$ & $\Delta t$ (days) \\
\hline 8P & 1.4 & Flare/Quiet & 360 & -0.25 \\
17P & $30-50$ & Flare/CIR & 400,700 & $5.2-7.4$ \\
$\mathrm{C} / 1999 \mathrm{~S} 4(\mathrm{LS} 4)$ & 3 & ICME & 592 & 1.09 \\
$\mathrm{C} / 1999 \mathrm{~T} 1(\mathrm{McH})$ & $6-20$ & CIR/Flare & 353 & 6.63 \\
2P/2003 & 0.7 & Flare/PS & 583 & -1.09 \\
9P/2005 & 0.9 & Quiet & 402 & -0.38 \\
\hline
\end{tabular}

abundances relative to $\mathrm{O}^{7+}$, but since $17 \mathrm{P}_{\text {had no }} \mathrm{O}^{7+}$ emission, we normalized its carbon and nitrogen emission to that of comet Linear S4 (hereafter LS4) and show these in Table 8. 17P encountered both a slow $400 \mathrm{~km} \mathrm{~s}^{-1}$ wind and a $700 \mathrm{~km} \mathrm{~s}^{-1}$ wind. Its very soft $\mathrm{X}$-ray spectrum, with little emission above $400 \mathrm{eV}$, is consistent with predictions made by Bodewits et al. 2007 for a very soft X-ray spectrum from a fast, cool, highlatitude wind. 17P has a much lower $\mathrm{C}^{5+}$ abundance than LS4, but its large $\mathrm{C}^{6+}$ ratio (38) is similar to comets that encountered solar winds with corotating interaction regions (CIRs) or flare events, consistent with the Ulysses solar wind observations. The $\mathrm{N}^{6+}$ ratio is much lower as expected for the soft spectrum of 17P and $\mathrm{Ne}^{9+}$ and $\mathrm{Ne}^{10+}$ values are limited by poor statistics.

$8 \mathrm{P}$ encountered a relatively slow solar wind of $\sim 360 \mathrm{~km} \mathrm{~s}^{-1}$ and its solar wind conditions are also summarized in Table 7. Its $\mathrm{O}^{8+}$ abundances for both the total and flare spectra are comparable to those of LS4 and $\mathrm{McH}$, although slightly lower. $8 \mathrm{P}^{\prime} \mathrm{S} \mathrm{C}^{6+}$ abundance is slightly higher than the LS4 values and nearly 50\% larger than the $\mathrm{McH}$ value. However, $8 \mathrm{P}$ has much larger $\mathrm{C}^{5+}$, with a value of $\approx 70$, which is more comparable to comets which encountered a solar wind that included a flare or CME. 8P's $\mathrm{N}^{6+}$ and $\mathrm{N}^{7+}$ abundances are also much larger than LS4 or McH's values are again more consistent with flare or CIR solar wind conditions. 8P's $\mathrm{Ne}^{10+}$ abundance is also larger, but limited by poor statistics.

\section{CONCLUSIONS}

We have presented results for the two most recent Chandra ACIS-S observations of comets made during solar minimum. Comet 17P/Holmes X-ray spectrum is unusually soft with little significant emission at energies above $500 \mathrm{eV}$. After careful background subtraction, we derive a $300-1000 \mathrm{eV}$ flux of $4.5 \times 10^{-13} \mathrm{erg} \mathrm{cm}^{-2} \mathrm{~s}^{-1}$, with over $90 \%$ of the emission in the $300-400 \mathrm{eV}$ range. The comet's X-rays are 3.5 from the nominal nucleus, and uncertainties in the comet's position from outgassing or other dynamical effects may be needed to explain this. However, we cannot distinguish between this significant excess emission and possible instrumental effects, such as incomplete charge transfer across the CCD. The lack of X-rays in the 400-1000 eV range may be attributed to the high latitude of 17P, and its interaction with the polar solar wind which is depleted in highly charged ions in accordance with model predictions for SWCX at high latitudes (Bodewits et al. 2007). 8P/Tuttle was much brighter than 17P, with an average count rate of 0.20 counts $\mathrm{s}^{-1}$ in the $300-1000 \mathrm{eV}$ range. We derive an average X-ray flux in this range of $9.4 \times$ $10^{-13} \mathrm{erg} \mathrm{cm}^{-2} \mathrm{~s}^{-1}$ and an X-ray luminosity for the comet of $1.7 \times 10^{14} \mathrm{erg} \mathrm{s}^{-1}$, one of the fainter comets observed with Chandra. The light curve showed a dramatic decrease in flux of over $60 \%$ between observations on January 1 and 4 . When comparing outer regions of the coma to inner regions, its spectra showed a decrease in ratios of $\mathrm{C} \mathrm{VI} / \mathrm{C} \mathrm{V}, \mathrm{O}$ VIII/ $\mathrm{O}$ VII, as predicted by recent SWCX emission models. There are remarkable differences between the X-ray emission from these two comets, further demonstrating the qualities of cometary $\mathrm{X}$-ray observations, and SWCX emission in general as a means of remote diagnostic of the interaction of astrophysical plasmas.

This work was supported by the Chandra X-ray Observatory's cycle 9 GO program, CXO-09100452, and archival program CXO-9100455. We thank Harvey Tannenbaum for Director's Discretionary Time to observe Comet 17P/Holmes (CXO-08108279), and we thank the Chandra X-ray Observatory's Scheduling and Mission Operations teams for executing these difficult and time-critical moving target observations. S.J.W. was supported by NASA contract NAS8-03060. D.J.C. thanks the California State University Northridge for support, and C.M.L. gratefully acknowledges support from Chandra GO program CXO-07108248. We also thank A. Fitzsimmons for useful discussions on 17P and Geronimo L. Villanueva for assistance in understanding the comet's orbital geometry. We are grateful for the cometary ephemerides of D. K. Yeomans published at the JPL/Horizons Web site.

\section{REFERENCES}

Arnaud, K. A. 1996, in ASP Conf. Ser. 101, Astronomical Data Analysis Software and Systems V, ed. G. H. Jacoby \& J. Barnes (San Francisco, CA: ASP), 17

Barber, R. J., Miller, S., Dello Russo, N., Mumma, M. J., Tennyson, J., \& Guio, P. 2009, MNRAS, 398, 1593

Beiersdorfer, P., et al. 2003, Science, 300, 1558

Bodewits, D., Hoekstra, R., Seredyuk, B., McCullough, R. W., Jones, G. H., \& Tielens, A. G. G. M. 2006, ApJ, 642, 593

Bodewits, D., Juhasz, Z., Hoekstra, R., \& Tielens, A. G. G. M. 2004, ApJ, 606, L81

Bodewits, D., et al. 2007, A\&A, 469, 1183

Table 8

Solar Wind Abundances for 8P and 17P Relative to $\mathrm{O}^{7+}$, Obtained from the SWCX-Model Fitting and Comparison to Linear S4 (LS4) and McNaight-Hartley (McH)

\begin{tabular}{lccccc}
\hline \hline Ion & 8P (full chip) & $8 \mathrm{P}$ (Int A) & $17 \mathrm{P}^{\mathrm{b}}$ & $\mathrm{LS} 4$ & $\mathrm{McH}$ \\
\hline $\mathrm{O}^{8+}$ & $0.28 \pm 0.05$ & $0.23 \pm 0.03$ & $<0.02$ & $0.32 \pm 0.03$ & $0.42 \pm 0.04$ \\
$\mathrm{C}^{6+}$ & $1.6 \pm 0.10$ & $0.71 \pm 0.08$ & $0.21 \pm 0.08$ & $1.4 \pm 0.4$ & $0.95 \pm 0.4$ \\
$\mathrm{C}^{5+}$ & $69 \pm 5$ & $77 \pm 5$ & $38 \pm 12$ & $12 \pm 4$ & $15 \pm 5$ \\
$\mathrm{~N}^{7+}$ & $0.25 \pm 0.03$ & $0.35 \pm 0.04$ & $0.024 \pm 0.018$ & $0.07 \pm 0.06$ & $0.19 \pm 0.06$ \\
$\mathrm{~N}^{6+}$ & $1.03 \pm 0.08$ & $1.20 \pm 0.12$ & $<0.16$ & $0.63 \pm 0.21$ & $0.47 \pm 0.20$ \\
$\mathrm{Ne}^{10+}$ & $0.04 \pm 0.03$ & $0.03 \pm 0.02$ & $<0.01$ & $0.02 \pm 0.01$ & $0.004 \pm 0.005$ \\
\hline
\end{tabular}

Notes.

${ }^{\text {a }}$ See the text for details.

${ }^{\mathrm{b}}$ Normalized 17P to LS4 $\mathrm{O}^{7+}$ line flux. 
Bonev, B. P., Mumma, M. J., Radeva, Y. L., DiSanti, M. A., Gibb, E. L., \& Villanueva, G. L. 2008, ApJ, 680, 61

Combi, M. R., Maekinen, J. T. T., Bertaux, J.-L., \& Ferron, S. 2008, IAU Circ., 8905,1

Cravens, T. E. 1997, Geophys. Res. Lett., 24, 105

Dennerl, K., Englhauser, J., \& Trümper, J. 1997, Science, 277, 1625

Fruscione, A., et al. 2006, Proc. SPIE, 6270, 60

Gaillard, B., Lecacheux, J., \& Colas, F. 2007, CBET, 1123

Harmon, J. K., Nolan, M. C., Howell, E. S., Giorgini, J. D., \& Magri, C. 2008, DPS, 40,501

Hsieh, H. H., Fitzsimmons, A., \& Pollacco, D. L. 2007, IAU Circ., 8897, 1

Kharchenko, V., \& Dalgarno, A. 2000, J. Geophys. Res., 105, 18351

Kharchenko, V., \& Dalgarno, A. 2001, ApJ, 554, L99

Kharchenko, V., Rigazio, M., Dalgarno, A., \& Krasnopolsky, V. A. 2003, ApJ, 858, L73

Krasnopolsky, V. A. 2004, J. Geophys. Res., 111, A12102

Krasnopolsky, V. A. 2006, Icarus, 67, 417

Krasnopolsky, V. A., Mumma, M. J., Abbott, M., Flynn, B. C., Meech, K. J., Yeomans, D. K., Feldman, P. D., \& Cosmovici, C. B. 1997, Science, 277, 1488
Lamy, P. L., Toth, I., Jorda, L., Weaver, H. A., Groussin, O., \& A'Hearn, M. F. 2008, DPS, 40, 502

Lisse, C. M., Christian, D. J., Dennerl, K., Meech, K. J., Petre, R., Weaver, H. A., \& Wolk, S. J. 2001, Science, 292, 1343

Lisse, C. M., et al. 1996, Science, 274, 205

Lisse, C. M., et al. 2005, ApJ, 635, 1329

McComas, D. J., Elliott, H. A., Schwadron, N. A., Gosling, J. T., Skoug, R. M., \& Goldstein, B. E. 2003, Geophys. Res. Lett., 30, 24

Montalto, M., Riffeser, A., Hopp, U., Wilke, S., \& Carraro, G. 2008, A\&A, 479, L45

Neugebauer, M., et al. 2000, J. Geophys. Res., 105, 20949

Pollacco, D., et al. 2006, PASP, 118, 1407

Salyk, C., et al. 2007, IAU Circ., 8890, 1

Schleicher, D. 2007, IAU Circ., 8889, 1

Schwadron, N. A., \& Cravens, T. E. 2000, ApJ, 544, 558

Sekanina, Z. 2008, ICQ, 30, 3

Voges, W., et al. 1999, A\&A, 349, 389

Wegmann, R., \& Dennerl, K. 2005, A\&A, 430, L33

Wegmann, R., Dennerl, K., \& Lisse, C. M. 2004, A\&A, 428, 647

Wolk, S. J., et al. 2009, ApJ, 694, 1293

Woodney, L., Schleicher, D. G., \& Bair, A. N. 2008, DPS, 40, 162 